\title{
SHP-2 positively regulates adipogenic differentiation in 3T3-L1 cells
}

\author{
TAKUYA UEHARA $^{1}$, KENJI SUZUKI ${ }^{1,2}$, HIDEYUKI YAMANAKA ${ }^{1}$, TAKAKO KIZAKI $^{2}$, TAKUYA SAKURAI $^{2}$, \\ YOSHINAGA ISHIBASHI ${ }^{2}$, HITOSHI ISHIDA ${ }^{3}$ and HIDEKI OHNO ${ }^{2}$ \\ ${ }^{1}$ Laboratory of Molecular Medicinal Sciences, Department of Bioscience and Bioinformatics, College of Information \\ Science and Engineering, Ritsumeikan University, Kusatsu, Shiga 525-8577; ${ }^{2}$ Department of Molecular Predictive \\ Medicine and Sport Science and ${ }^{3}$ Third Department of Internal Medicine, Kyorin University, \\ School of Medicine, Mitaka, Tokyo 181-8611, Japan
}

Received February 5, 2007; Accepted March 16, 2007

\begin{abstract}
The Src homology domain 2 (SH2)-containing tyrosine phosphatase SHP-2 has been implicated in the regulation of proliferation and differentiation in various cell types. Here, we investigated the ability of SHP-2 to mediate insulin-induced adipogenic differentiation of mouse 3T3-L1 cells. We found that the expression of SHP-2 was increased along with adipogenic differentiation. Overexpression of wild-type SHP-2 in 3T3-L1 cells resulted in enhanced adipocyte differentiation. Furthermore, insulin-stimulated adipogenic differentiation of 3T3-L1 cells was abolished by downregulating SHP-2 expression using short interfering RNA. These results suggest that SHP-2 is a positive effector in signal transduction pathways necessary for adipocyte differentiation. In SHP-2 knockdown cells, the expression of peroxisome proliferator-activated receptor $\gamma$, a master regulator of adipogenesis, was entirely suppressed even in the late phase of differentiation, whereas the expression level of C/EBP $\delta$ was unchanged. These results highlight a novel role of SHP-2 in the signal transduction pathways regulating adipocyte differentiation.
\end{abstract}

\section{Introduction}

Obesity is a major risk factor for diseases such as type-2 diabetes, cardiovascular disease, stroke, and hypertension. As a specialized organ for energy storage, adipocytes synthesize and store triglycerides during periods of caloric excess, and they mobilize these energy depots as free fatty acids and glycerol during periods of nutritional deprivation (1). Obesity

Correspondence to: Dr Kenji Suzuki, Laboratory of Molecular Medicinal Sciences, Department of Bioscience and Bioinformatics, College of Information Science and Engineering, Ritsumeikan University, Kusatsu, Shiga 525-8577, Japan

E-mail: kenjiszk@is.ritsumei.ac.jp

Key words: SHP-2, adipogenesis, protein tyrosine phosphatases, signal transduction, 3T3-L1 cells is the result of an overgrowth of adipose tissue due to an imbalance between energy intake and energy expenditure. At the cellular level, adipose tissue overgrowth is associated with adipocyte hypertrophy and hyperplasia $(2,3)$. Hypertrophy results from excessive triglyceride accumulation in adipocytes, whereas hyperplasia results from recruitment of new adipocytes via the proliferation and differentiation of preadipocytes.

Although adipose tissue has long been considered a passive tissue specialized in energy storage, the concept of adipose tissue as an energy warehouse has been recently revised (4). Increasing evidence indicates that adipose tissue is an important endocrine organ that secretes adipocytokines, such as leptin and adiponectin, which modulate lipid and carbohydrate metabolism. Adipose tissue plays a central role in whole body energy homeostasis $(5,6)$. In view of the prevalence of obesity and obesity-related diseases, it is important to identify factors regulating adipocyte development and function.

Several key transcription factors have been reported to be involved in adipocyte differentiation, including peroxisome proliferator-activated receptor $\gamma(\mathrm{PPAR} \gamma)$, CCAAT/enhancerbinding protein $(\mathrm{C} / \mathrm{EBP})$ family proteins, and adipocyte determination-differentiation factor 1 (7-10). These transcription factors are induced at different stages of adipocyte differentiation, and they functionally interact with each other to carry out adipogenesis and lipogenesis by regulating the expression of various genes $(11,12)$. The upstream signals regulating the induction and expression of these transcription factors, however, are poorly understood.

Many external hormones such as insulin, insulin-like growth factor-1, and glucocorticoids are known to promote the proliferation and/or differentiation of preadipocytes $(13,14)$. Of these, insulin is well known to play a key role in adipocyte differentiation in vivo and in vitro. Insulin elicits diverse biological actions such as proliferation and differentiation by binding to its tyrosine kinase receptors. Upon ligand binding, the insulin receptor dimerizes and autophosphorylates on tyrosine residues. This in turn stimulates the phosphorylation of a number of intracellular substrates. These molecules, including the insulin receptor substrate 
(IRS) family, Shc, Gab1, and Grb10, act as adaptor proteins that transduce signals from the cell surface receptor to downstream effectors (15-17). The roles of protein tyrosine phosphatases (PTPs) in signal transduction have been examined for several growth factor receptors. SHP-2, a member of the PTP family, is ubiquitously expressed and generally positively regulates signaling, for example, by the epidermal growth factor (EGF) and platelet-derived growth factor (PDGF) receptors (18-21). Tyrosine phosphorylated SHP-2 acts as an adaptor molecule that recruits Grb2 and other proteins, leading to the activation of mitogen-activated protein kinases (MAPKs) downstream of the several growth factor receptors $(22,23)$.

To better understand the molecular mechanism of how insulin regulates adipogenesis via downstream signaling molecules, we investigated the role of SHP-2 in adipocyte differentiation by 3T3-L1 cells. We found that overexpression of SHP-2 in these cells results in enhanced adipocyte differentiation. Furthermore, insulin-stimulated adipogenic differentiation of 3T3-L1 cells was abolished by downregulating SHP-2 expression using a short interfering RNA. These results highlight a novel role of SHP-2 in the signal transduction pathways regulating adipocyte differentiation.

\section{Materials and methods}

Antibodies and reagents. Monoclonal antibodies against SHP-2 and GAPDH were purchased from Santa Cruz Biotechnology. Dulbecco's modified Eagle's medium (DMEM) was from Sigma. TRIzol reagents and G418 were from Invitrogen. GeneJammer transfection reagent was from Stratagene.

Cell culture. 3T3-L1 cells were grown in DMEM containing heat-inactivated $10 \%$ fetal bovine serum (FBS) at $37^{\circ} \mathrm{C}$ in an atmosphere containing $5 \% \mathrm{CO}_{2}$. Cells were used in differentiation experiments 2 days after they reached confluence. Adipocyte differentiation was induced by treating the cells with medium containing $1 \mu \mathrm{g} / \mathrm{ml}$ insulin, $1 \mu \mathrm{M}$ dexamethasone, $1 \mathrm{mM}$ isobutyl-methylxanthine, and 10\% FBS. After 2 days, the differentiation medium was replaced with post-differentiation medium containing $1 \mu \mathrm{g} / \mathrm{ml}$ insulin and $10 \%$ FBS. After an additional 2 days, the medium was switched back to DMEM supplemented with $10 \%$ FBS.

Oil Red $O$ staining. 3T3-L1 cells were washed with phosphate-buffered saline and $60 \%$ isopropyl alcohol and then stained with filtered Oil Red O staining solution $(0.3 \%$ Oil Red $\mathrm{O}$ in $60 \%$ isopropyl alcohol) for $15 \mathrm{~min}$ at $37^{\circ} \mathrm{C}$.

Stable expression of SHP-2. The coding region of wild-type human SHP-2 was subcloned in pcDNA3.1/myc-His (Invitrogen). To generate an SHP-2 short hairpin RNA (shSHP-2), a pair of 59-bp oligonucleotides (5'-GATCCGC ACAGTACCGGTTTATCTTTCAAGAGAAGATAAACCG GTACTGTGCTTTTTTT-3' and 5'-CTAGAAAAAAAGC ACAGTACCGGTTTATCTTCTCTTGAAAGATAAACC GGTACTGTGCG-3) were synthesized, annealed, and ligated between the BamHI and XbaI sites of pGE-1 (Stratagene). The expressed hairpin is complementary to nucleotides 1685-1703 of the mouse SHP-2 mRNA sequence. Constructs were verified by restriction analysis and sequencing. 3T3-L1 cells stably expressing hSHP-2 or a small interfering RNA (siRNA) targeting mouse SHP-2 were prepared by transfecting the cells with the constructs and then growing them in selection medium containing $0.8 \mathrm{mg} /$ $\mathrm{ml} \mathrm{G}-418$. Gene expression and functional analysis were performed on individual G-418-resistant clones.

$R N A$ preparation and quantitative real-time PCR. Total RNA was isolated from 3T3-L1 cells using TRIzol reagent (Invitrogen) according to the manufacturer's instructions. After quantification by spectrophotometry, $2 \mu \mathrm{g}$ of RNA was reverse-transcribed into cDNA at $42^{\circ} \mathrm{C}$ for 45 min using an ExScript RT reagent kit (Takara) in the presence of the random hexamer. Quantitative RT-PCR was performed using a Platinum SYBR-Green qPCR SuperMix UDG (Invitrogen). The oligonucleotides used for analysis of mouse gene expression were as follows: mouse SHP-2, 5'-CATGCCTG AGTTTGAGACCA-3' (sense) and 5'-TCCGACCTTAGAG AGTTTGA-3' (antisense); hSHP-2, 5'-CGGTCTGGCAATA CCACTTT-3' (sense) and 5'-GATATCTCTGAAACTTT TCTGCTGTTGC-3' (antisense); PPAR $\gamma$, 5'-AGACAACG GACAAATCACCAT-3' (sense) and 5'-TCACAGCAAACT CAAACTTAGG-3' (antisense); fatty acid-binding protein (FABP), 5'-CCAGTGAAAACTTCGATGATTAC-3' (sense) and 5'-CATTCCACCACCAGCTTGTCACC-3' (antisense); lipoprotein lipase, 5'-CGAAATTTCTCTGTACGGCAC-3' (sense) and 5'-CTGCAGATGAGAAACTTTCT-3' (antisense); C/EBPo, 5'-CCAGGAGATGCAGCAGAAG-3' (sense) and 5'-GGGGTCTGAGGTATAGGTCG-3' (antisense); Pref-1, 5'-TCTGCGAGGCTGACAATGTCTGC-3' (sense) and 5'CCTTGTGCTGGCAGTCCTTTCC-3' (antisense); and cyclophilin, 5'-AGCACTGGAGAGAAAGGATT-3' (sense) and 5'-CACAATGTTCATGCCTTCTT-3' (antisense).

Western blot analysis. Cells were homogenized in sample buffer (62.5 mM Tris-HCl, pH 6.8, 2\% SDS, $5 \mathrm{mg} / \mathrm{ml}$ bromphenol blue, and $10 \%$ glycerol), and proteins were separated by SDS-PAGE on a $10 \%$ acrylamide gel and then transferred to a PVDF membrane. The membrane was incubated for $1 \mathrm{~h}$ at room temperature in TBS/ $0.05 \%$ Tween-20 containing $5 \%$ dry-milk and then for $1.5 \mathrm{~h}$ in TBS $/ 0.05 \%$ Tween-20 containing a mouse monoclonal antibody raised against a C-terminal SHP-2 peptide. Immunostained proteins were visualized with HRP-conjugated anti-mouse IgG (Cappel), followed by Western blotting luminol reagent (Santa Cruz Biotechnology).

Statistical analysis. Data were expressed as mean \pm SE of values from several experiments, and statistical significance was evaluated using the unpaired t-test. A value of $\mathrm{p}<0.05$ was considered statistically significant.

\section{Results and discussion}

Insulin is an important metabolic hormone secreted by pancreatic $\beta$ cells that promotes the synthesis and storage of carbohydrates, lipids, and proteins and inhibits their degradation and release into circulation. Insulin action is 


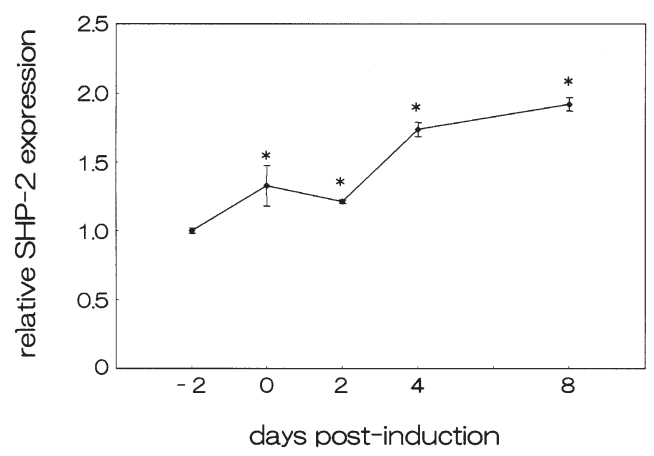

Figure 1. Expression of SHP-2 is up-regulated by adipogenic differentiation. 3T3-L1 cells were differentiated into adipocytes, and total RNA was prepared at the indicated stage of differentiation. The expression of SHP-2 was measured by RT-PCR. " $\mathrm{P}<0.05$ vs. undifferentiated 3T3-L1 cells.

A

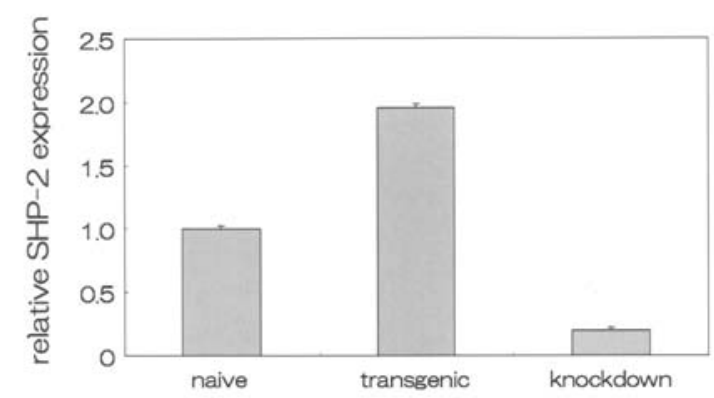

B

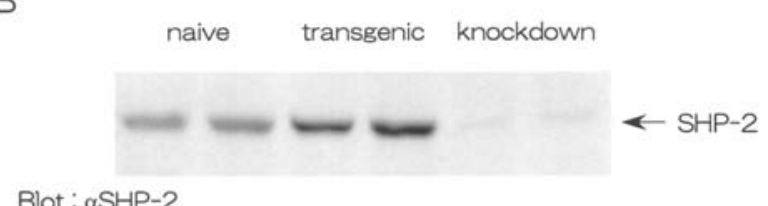

Blot : $\alpha$ SHP-2

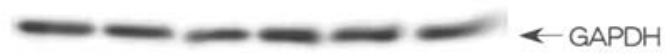

Blot : $\alpha \mathrm{GAPDH}$

Figure 2. Establishment of SHP-2 transgenic and knockdown cell lines. (A) Total RNA was prepared, and the expression of SHP-2 was measured by RT-PCR. (B) Cell extracts were prepared and analyzed by Western blotting using antibodies against SHP-2 and GAPDH.

mediated, in part, by the activation of protein kinases and phosphatases, which modulate transcription and promote cellular growth and differentiation. Insulin is generally thought to play a crucial role in the process of adipogenesis (24-27). To determine whether SHP-2 is involved in this process, we first examined the expression pattern of SHP-2 during adipocyte differentiation in 3T3-L1 cells by real-time RT-PCR (Fig. 1). On day 4 of differentiation, the level of SHP-2 expression was markedly increased.

To examine whether SHP-2 influences adipogenesis, we established 3T3-L1 cells overexpressing human SHP-2 and cells in which SHP-2 was knocked down using an siRNA. The expression of mRNA and protein for mouse and/or human SHP-2 was assessed by RT-PCR (Fig. 2A) and Western blotting (Fig. 2B), respectively, and the differentiation status was examined by staining with Oil Red $\mathrm{O}$ and by measuring the level of mRNAs encoding adipose-specific genes such as PPAR $\gamma$ and FABP. Treatment of the cells overexpressing wild-type SHP-2 with differentiation medium resulted in an increase in the accumulation of intracellular lipid droplets compared to naïve (untransfected) cells (Fig. 3). On the other hand, no significant lipid accumulation occurred in cells expressing the SHP-2 siRNA. These results indicate that SHP-2 is a positive regulator of 3T3-L1 adipogenesis.

To elucidate the role of SHP-2 in adipocyte differentiation, we next examined the effects of knocking down SHP-2 on the expression of adipocyte-specific genes (Fig. 4). In naïve 3T3-L1 cells, the expression of Pref-1, a preadipocytesecreted factor that inhibits adipogenesis, was gradually decreased as the cells differentiated, which agrees with the findings of previous studies (28). Suppression of SHP-2 expression increased the expression of Pref- 1 without inducing adipogenesis. A similar pattern of expression was observed in both control and SHP-2-depleted cells for $\mathrm{C} / \mathrm{EBP}$, which is induced in the early phase of adipogenesis, although its expression in SHP-2 knockdown cells was upregulated on day 8 of differentiation. On the other hand, the induction of PPAR $\gamma$ was completely suppressed in the SHP-2 knockdown cells. Expression of FABP, another adipocytespecific protein, was also suppressed in SHP-2-depleted cells. These results support the idea that SHP-2 positively regulates adipocyte differentiation.

The phosphorylation of proteins on tyrosine residues serves as a switch regulating various cellular events as well as the cellular response to environmental stimuli. Tyrosine phosphorylation is controlled by the coordinated actions of protein tyrosine kinases and PTPs. PTPs play both inhibitory and permissive roles in the physiological responses to particular ligands, and SHP-2 has been shown to participate in the activation of MAPKs by EGF (29). SHP-2 binds directly to activated PDGF and EGF receptors via its $\mathrm{N}$ terminal SH2 domain. SHP-2 also plays a pivotal role in insulin signaling related to cellular proliferation and differentiation. Our results suggested that SHP-2 plays a positive role in insulin-induced adipocyte differentiation by 3T3-L1 cells.

Adipocyte differentiation is a complex process that requires communication between intracellular signaling cascades evoked by extracellular stimuli such as insulin and transcription factors in the nucleus (30). The molecular mechanism regulating the transcription of adipocyte-specific genes during the process of preadipocyte differentiation is relatively well characterized (31). On the other hand, the intracellular signaling pathways downstream of the insulin receptor tyrosine kinase are not completely understood. Upon ligand binding, the insulin receptor phosphorylates itself as well as cytosolic proteins including IRS proteins and Shc $(15,16,32)$. These phosphorylated proteins then recruit other $\mathrm{SH} 2$ domain-containing signaling proteins such as the p85 subunit of phosphatidylinositol 3-kinase (PI3K), Grb2, and SHP-2 (33-35). Activation of the PI3K pathway is necessary 
naive

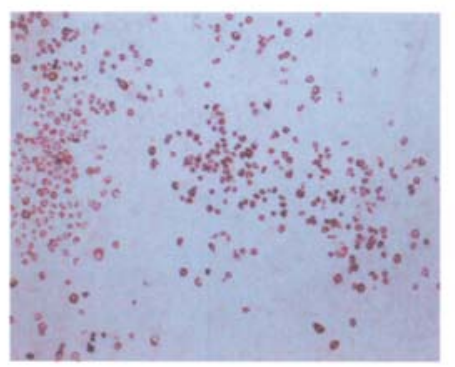

SHP-2 transgenic

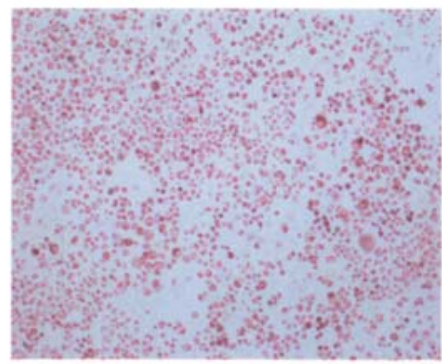

SHP-2 knockdown

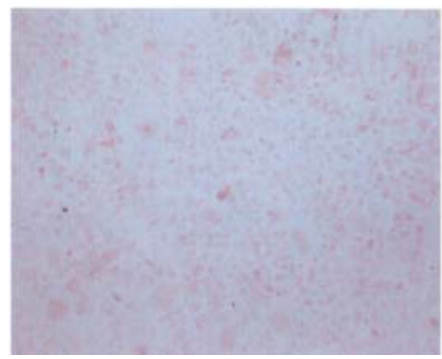

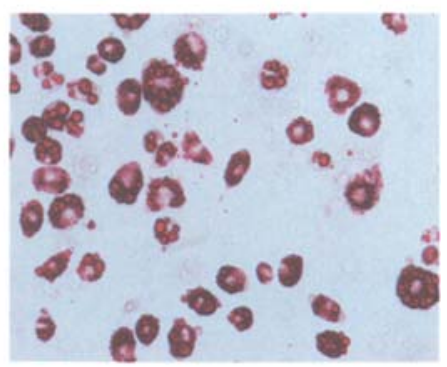
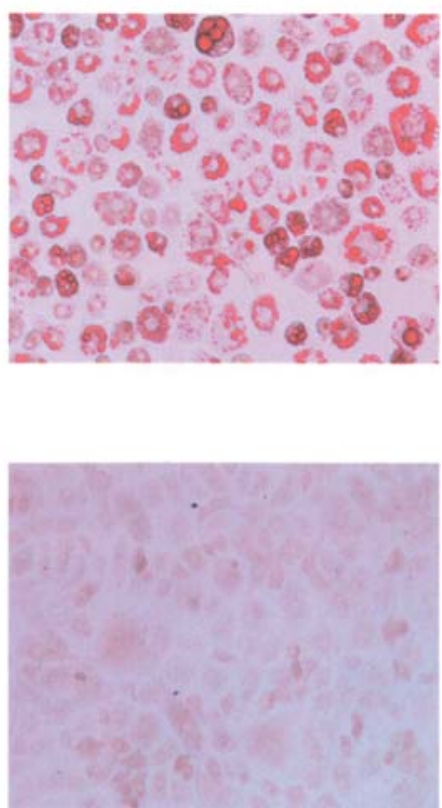

Figure 3. Expression of SHP-2 influences adipocyte differentiation. Naïve, SHP-2 overexpressing, and SHP-2 suppressed 3T3-L1 cells were differentiated into adipocytes. On day 8, the cells were stained with Oil Red O.

A Pref-1

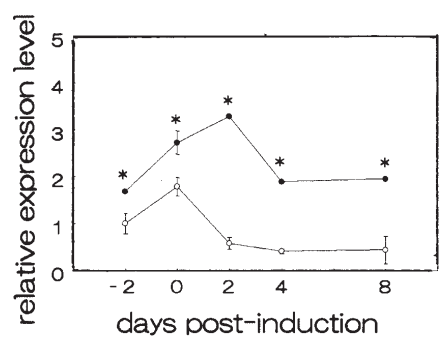

C PPAR

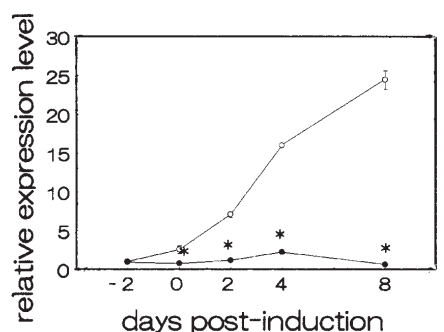

$\mathrm{B}$ C/EBP $\delta$

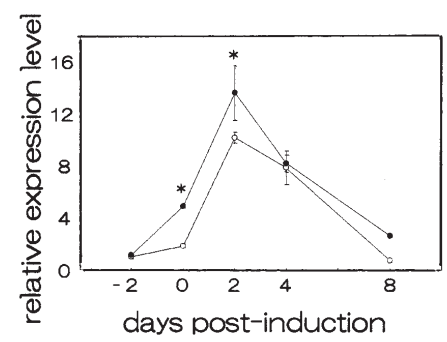

D FABP

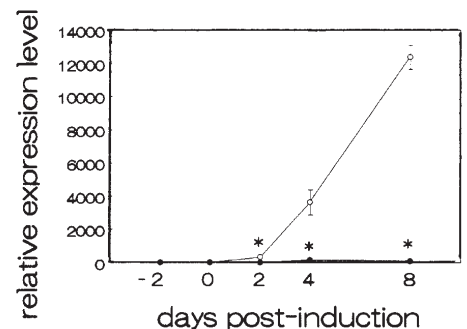

Figure 4. SHP-2 knockdown suppresses adipogenic differentiation. Adipocyte differentiation was induced in naïve (๑) and SHP-2 knockdown (•) 3T3-L1 cells, and total RNA was prepared from the cells at the indicated stage of differentiation. Expression of Pref-1 (A), C/EBPo (B), PPAR (C), and FABP (D) were measured by RT-PCR. "P<0.05 vs. undifferentiated naïve 3T3-L1 cells. 
for complete differentiation of preadipocytes, and the binding of IRS- 1 and IRS-2 to PI3K is transiently increased during adipocyte differentiation (36). Because SHP-2 participates in growth factor activation of PI3K, SHP-2 is thought to mediate insulin-induced adipocyte differentiation in 3T3-L1 cells by activating PI3K (37).

MAPK activation is known to be required for the mitogenic response, but the role for MAPK in the regulation of cellular differentiation is not fully understood. Some studies in PC12 cells have suggested that the sustained activation of the MAPK cascade induces neuronal differentiation, but other studies have found that this prolonged activation of MAPK is not sufficient for their terminal differentiation $(38,39)$. The role of MAPK activation in adipocyte differentiation is also controversial. Studies with antisense RNAs by Sale et al have indicated that MAPK activation is necessary for insulininduced adipocyte differentiation of 3T3-L1 cells (40). On the other hand, Porras et al showed that MAPK inhibits insulin-induced differentiation in rat fetal brown adipocytes (41). In addition, MAPK-mediated phosphorylation of PPAR $\gamma$, a master regulator of adipocyte differentiation, suppresses its transcriptional activity, leading to the inhibition of adipogenesis (42).

There is also some disagreement regarding the role of SHP-2 in tyrosine kinase signaling and the relative contributions of its $\mathrm{SH} 2$ domains, catalytic domain, and Cterminal tail in its downstream effects. Two tyrosine phosphorylation sites located at the C-terminus of SHP-2 are consensus binding sites for the SH2 domain of the Grb2 adaptor protein (43). Thus, SHP-2 may act as a positive effector of signal transduction by serving as an adaptor protein linking Grb2 and mSos to Ras, leading to activation of the MAPK cascade. Conversely, SHP-2 has been demonstrated to function as a negative regulator in the interferonstimulated JAK/STAT pathway, resulting in cell growth arrest (44). In this case, specific substrate proteins of SHP-2 might be involved. Alternatively, tyrosine residues at the Cterminal tail of SHP-2 may play an important role in balancing cell growth and differentiation. How SHP-2 regulates insulin intracellular signaling pathways downstream of the insulin receptor remains to be determined.

In conclusion, we have demonstrated that SHP-2 plays a positive role in adipocyte differentiation of 3T3-L1 cells. The precise mechanism of how SHP-2 regulates the differentiation process remains unknown, but it may act at the early stage of the differentiation. Further studies are needed to define the signaling events downstream of SHP-2 that are responsible for adipocyte differentiation and to determine how the various adipogenic signals influence each other during the differentiation process.

\section{References}

1. Hwang CS, Loftus TM, Mandrup S and Lane MD: Adipocyte differentiation and leptin expression. Annu Rev Cell Dev Biol 13: 231-259, 1997.

2. Hirsch J and Batchelor B: Adipose tissue cellularity in human obesity. Clin Endocrinol Metab 5: 299-311, 1976.

3. Kras KM, Hausman DB, Hausman GJ and Martin RJ: Related articles, adipocyte development is dependent upon stem cell recruitment and proliferation of preadipocytes. Obes Res 7: 491-497, 1999.
4. Spiegelman BM and Flier JS: Obesity and the regulation of energy balance. Cell 104: 531-543, 2001.

5. Ahima RS and Flier JS: Adipose tissue as an endocrine organ. Trends Endocrinol Metab 11: 327-332, 2000.

6. Trayhurn P and Beattie JH: Physiological role of adipose tissue: white adipose tissue as an endocrine and secretory organ. Proc Nutr Soc 60: 329-339, 2001.

7. Tontonoz P, Hu E, Graves RA, Budavari AI and Spiegelman BM: mPPAR gamma 2: tissue-specific regulator of an adipocyte enhancer. Genes Dev 8: 1224-1234, 1994.

8. Christy RJ, Yang VW, Ntambi JM, Geiman DE, Landschulz WH, Friedman AD, Nakabeppu Y, Kelly TJ and Lane MD: Differentiation-induced gene expression in 3T3-L1 preadipocytes: CCAAT/enhancer binding protein interacts with and activates the promoters of two adipocyte-specific genes. Genes Dev 3: 1323-1335, 1989.

9. Freytag SO and Geddes TJ: Reciprocal regulation of adipogenesis by Myc and C/EBP alpha. Science 256: 379-382, 1992 .

10. Wu Z, Xie Y, Bucher NL and Farmer SR: Conditional ectopic expression of C/EBP beta in NIH-3T3 cells induces PPAR gamma and stimulates adipogenesis. Genes Dev 9: 2350-1363, 1995.

11. Tontonoz P, Kim JB, Graves RA and Spiegelman BM: ADD1: a novel helix-loop-helix transcription factor associated with adipocyte determination and differentiation. Mol Cell Biol 13: 4753-4759, 1993.

12. Kim JB and Spiegelman BM: ADD1/SREBP1 promotes adipocyte differentiation and gene expression linked to fatty acid metabolism. Genes Dev 10: 1096-1107, 1996.

13. Ailhaud G, Grimaldi P and Negrel R: Cellular and molecular aspects of adipose tissue development. Annu Rev Nutr 12: 207-233, 1992.

14. Smas CM and Sul HS: Control of adipocyte differentiation. Biochem J 309: 697-710, 1995.

15. White MF: The IRS-signalling system: a network of docking proteins that mediate insulin action. Mol Cell Biochem 182: 3-11, 1998.

16. Sasaoka T, Rose DW, Jhun BH, Saltiel AR, Draznin B and Olefsky JM: Evidence for a functional role of Shc proteins in mitogenic signaling induced by insulin, insulin-like growth factor-1, and epidermal growth factor. J Biol Chem 269: 13689-13694, 1994.

17. Holgado-Madruga M, Emlet DR, Moscatello DK, Godwin AK and Wong AJ: A Grb2-associated docking protein in EGF- and insulin-receptor signalling. Nature 379: 560-564, 1996.

18. Noguchi T, Matozaki T, Horita K, Fujioka Y and Kasuga M: Role of SH-PTP2, a protein-tyrosine phosphatase with Src homology 2 domains, in insulin-stimulated Ras activation. Mol Cell Biol 14: 6674-6682, 1994.

19. Yamauchi K, Milarski KL, Saltiel AR and Pessin JE: Proteintyrosine-phosphatase SHPTP2 is a required positive effector for insulin downstream signaling. Proc Natl Acad Sci USA 92: 664-668, 1995.

20. Bennett AM, Hausdorff SF, O'Reilly AM, Freeman RM and Neel BG: Multiple requirements for SHPTP2 in epidermal growth factor-mediated cell cycle progression. Mol Cell Biol 16: 1189-1202, 1996.

21. Shi ZQ, Lu W and Feng GS: The Shp-2 tyrosine phosphatase has opposite effects in mediating the activation of extracellular signal-regulated and c-Jun NH2-terminal mitogen-activated protein kinases. J Biol Chem 273: 4904-4908, 1998.

22. Li W, Nishimura R, Kashishian A, Batzer AG, Kim WJ, Cooper JA and Schlessinger J: A new function for a phosphotyrosine phosphatase: linking GRB2-Sos to a receptor tyrosine kinase. Mol Cell Biol 14: 509-517, 1994.

23. Bazenet CE, Gelderloos JA and Kazlauskas A: Phosphorylation of tyrosine 720 in the platelet-derived growth factor alpha receptor is required for binding of Grb2 and SHP-2 but not for activation of Ras or cell proliferation. Mol Cell Biol 16: 6926-6936, 1996.

24. Rubin CS, Hirsch A, Fung C and Rosen OM: Development of hormone receptors and hormonal responsiveness in vitro. Insulin receptors and insulin sensitivity in the preadipocyte and adipocyte forms of 3T3-L1 cells. J Biol Chem 253: 7570-7578, 1978.

25. Chapman AB, Knight DM, Diecjmann BS and Ringold GM: Analysis of gene expression during differentiation of adipogenic cells in culture and hormonal control of the developmental program. J Biol Chem 259: 15548-15555, 1984. 
26. Moustaid N, Lasnier F, Hainque B, Quignard-Boulange A and Pairault J: Analysis of gene expression during adipogenesis in 3T3-F442A preadipocytes: insulin and dexamethasone control. J Cell Biochem 42: 243-254, 1990.

27. Suryawan A, Swanson LV and Hu CY: Insulin and hydrocortisone, but not triiodothyronine, are required for the differentiation of pig preadipocytes in primary culture. J Anim Sci 75: 105-111, 1997.

28. Smas CM and Sul HS: Pref-1, a protein containing EGF-like repeats, inhibits adipocyte differentiation. Cell 73: 725-734, 1993.

29. Qu CK, Yu WM, Azzarelli B and Feng GS: Genetic evidence that Shp-2 tyrosine phosphatase is a signal enhancer of the epidermal growth factor receptor in mammals. Proc Natl Acad Sci USA 96: 8528-8533, 1999.

30. Rosen ED and Spiegelman BM: Molecular regulation of adipogenesis. Annu Rev Cell Dev Biol 16: 145-171, 2000.

31. Rangwala SM and Lazar MA: Transcriptional control of adipogenesis. Annu Rev Nutr 20: 535-559, 2000

32. Pronk GJ, McGlade J, Pelicci G, Pawson T and Bos JL: Insulininduced phosphorylation of the $46-$ and $52-\mathrm{kDa}$ Shc proteins. J Biol Chem 268: 5748-5753, 1993.

33. Kuhne MR, Pawson T, Lienhard GE and Feng GS: The insulin receptor substrate 1 associates with the $\mathrm{SH} 2$-containing phosphotyrosine phosphatase SHp. J Biol Chem 268: 11479$11481,1993$.

34. Baltensperger K, Kozma LM, Cherniack AD, Klarlund JK, Chawla A, Banerjee U and Czech MP: Binding of the Ras activator son of sevenless to insulin receptor substrate-1 signaling complexes. Science 260: 1950-1952, 1993.

35. Okada T, Kawano Y, Sakakibara T, Hazeki O and Ui M: Essential role of phosphatidylinositol 3-kinase in insulininduced glucose transport and antilipolysis in rat adipocytes. Studies with a selective inhibitor wortmannin. J Biol Chem 269: 3568-3573, 1994.
36. Sakaue H, Ogawa W, Matsumoto M, Kuroda S, Takata M Sugimoto T, Spiegelman BM and Kasuga M: Posttranscriptional control of adipocyte differentiation through activation of phosphoinositide 3-kinase. J Biol Chem 273: 28945-28952, 1998 .

37. Wu CJ, O'Rourke DM, Feng GS, Johnson GR, Wang Q and Greene MI: The tyrosine phosphatase SHP-2 is required for mediating phosphatidylinositol 3-kinase/Akt activation by growth factors. Oncogene 20: 6018-6025, 2001.

38. Cowley S, Paterson H, Kemp P and Marshall CJ: Activation of MAP kinase kinase is necessary and sufficient for PC12 differentiation and for transformation of NIH 3 T3 cells. Cell 77: 841-852, 1994

39. Font de Mora J, Porras A, Ahn N and Santos E: Mitogenactivated protein kinase activation is not necessary for, but antagonizes, 3T3-L1 adipocytic differentiation. Mol Cell Biol 17: 6068-6075, 1997.

40. Sale EM, Atkinson PG and Sale GJ: Requirement of MAP kinase for differentiation of fibroblasts to adipocytes, for insulin activation of p90 S6 kinase and for insulin or serum stimulation of DNA synthesis. EMBO J 14: 674-684, 1995.

41. Porras A, Alvarez AM, Valladares A and Benito M: p42/p44 mitogen-activated protein kinase activation is required for the insulin-like growth factor-I/insulin induced proliferation, but inhibits differentiation, in rat fetal brown adipocytes. Mol Endocrinol 12: 825-834, 1998

42. Hu E, Kim JB, Sarraf P and Spiegelman BM: Inhibition of adipogenesis through MAP kinase-mediated phosphorylation of PPARgamma. Science 274: 2100-2103, 1996.

43. Vogel W and Ullrich A: Multiple in vivo phosphorylated tyrosine phosphatase SHP-2 engages binding to Grb2 via tyrosine 584. Cell Growth Differ 7: 1589-1597, 1996.

44. You M, Yu DH and Feng GS: Shp-2 tyrosine phosphatase functions as a negative regulator of the interferon-stimulated Jak/STAT pathway. Mol Cell Biol 19: 2416-2424, 1999. 